\title{
Mitochondrial Genome Recombination in Somatic Hybrids of Solanum Commersonii and S. Tuberosum
}

\section{Kwang-Soo Cho ( $\square$ kscholvoe@korea.kr)}

National Institute of Crop Science, Rural Development Administration

Hyun-Oh Lee

Seoul National University

Sang-Choon Lee

Phyzen Genomics Institute

Hyun-Jin Park

National Institute of Crop Science, Rural Development Administration

\section{Jin-Hee Seo}

National Institute of Crop Science, Rural Development Administration

Ji-Hong Cho

National Institute of Crop Science, Rural Development Administration

Young-Eun Park

National Institute of Crop Science, Rural Development Administration

Jang-Gyu Choi

National Institute of Crop Science, Rural Development Administration

Tae-Jin Yang

Seoul National University

\section{Research Article}

Keywords: Solanum tuberosum, Solanum commersonii, somatic hybrid, mitochondrial genome, mitogenome, homologous recombination

Posted Date: December 15th, 2021

DOI: https://doi.org/10.21203/rs.3.rs-1091981/v1

License: (c) (i) This work is licensed under a Creative Commons Attribution 4.0 International License. Read Full License 


\section{Abstract}

Interspecific somatic hybridization has been performed in potato breeding experiments to increase plant resistance against biotic and abiotic stress conditions. We analyzed the mitochondrial and plastid genomes and $45 S$ nuclear ribosomal DNA (45S rDNA) for the cultivated potato ( $S$. tuberosum, St), wild potato (S. commersonii, Sc), and their somatic hybrid (StSc). Complex genome components and structure, such as the hybrid form of $45 \mathrm{~S}$ rDNA in StSc, unique plastome in Sc, and recombinant mitogenome were identified. However, the mitogenome exhibited dynamic multipartite structures in both species as well as in the somatic hybrid. In St, the mitogenome is 756,058 bp and is composed of five subgenomes ranging from 297,014 to $49,171 \mathrm{bp}$ in St. In Sc, it is 552,103 bp long and is composed of two sub-genomes of 338,427 and 213,676 bp length. StSc has 447,645 bp long mitogenome with two subgenomes of length 398,439 and 49,206 bp. The mitogenome structure exhibited dynamic recombination mediated by tandem repeats; however, it contained highly conserved genes in the three species. Among the 35 protein-coding genes of the StSc mitogenome, 21 were identical for all the three species, and 12 and 2 were unique in Sc and St, respectively. The recombinant mitogenome might be derived from homologous recombination between both species during somatic hybrid development.

\section{Introduction}

Potato (Solanum tuberosum) belongs to Solanaceae and is the fourth important food crop for human consumption worldwide. Plant cytoplasmic genomes, such as plastid (plastomes) and mitochondrial (mitogenomes) genomes, are maternally inherited and are usually present in high copy numbers in plant cells ${ }^{1}$. The mitochondrion independently duplicates its genome and governs the cell energy supply and plant development ${ }^{2}$. The mitogenome exists as a mixture of variable sizes mediated by recombination between tandem repeat sequences ${ }^{3}$, while plastomes are highly conserved in their variation and size ${ }^{4}$. High levels of recombination and foreign DNA integration result in most of the size variation in mitogenomes, and variable forms of mitochondrial gene transcripts occur due to multiple transcript initiation and termination sites, trans-splicing, and RNA editing ${ }^{4-7}$. The intraspecific structural variation of the mitogenome has been found to be extraordinarily high in plant species, such as the multipartite forms in Silene species ${ }^{8}$.

While cytoplasmic genetic elements are maternally inherited in most plants, their inheritance patterns are quite different in somatic hybrids generated by conducting the protoplast fusion. Somatic hybrids carry plastomes derived from one of the two parents without sequence variation or rearrangement. However, mitogenome maintenance has not yet been reported in somatic hybrids. A previous study suggested that mitogenomes might be presented as fused forms by finding a novel fingerprinting band pattern in the somatic hybrid progenies of Solanum melongena cv. Black Beauty and Solanum torvum ${ }^{9}$.

Complete mitogenome sequences have been released from over 11,354 species of animals, fungi, plants, and protists (Organelle Genome Resources, https://www.ncbi.nlm.nih.gov/genome/organelle/), which has facilitated research on the genetic and evolutionary nature of mitogenomes ${ }^{10-12}$. Mitogenome sequences were released for crop species in Solanaceae including pepper (Capsicum annum), potato $(S$. tuberosum), and tomato (S. lycopersicum) ${ }^{13-15}$, and mitogenome structures and phylogenetic relationships among these species have been revealed. Previously, we reported the complete mitogenome sequences for St and Sc; however, a detailed genome analysis was not conducted ${ }^{14,16}$.

Somatic hybridization mediated by protoplast fusion can be a useful breeding tool for introducing valuable traits from related species ${ }^{17-19}$. Favorable traits such as disease resistance ${ }^{20,21}$ and drought tolerance ${ }^{22}$ were transmitted to the cultivated potato from wild species. Similar approaches have been applied to develop potato somatic hybrids with enhanced disease resistance using wild potato species ${ }^{23-27}$. The organelle genome structures in somatic hybrids have been investigated in these studies. Conserved intact plastomes were delivered to the somatic hybrid from either of the two parents. Novel mitogenomes have been identified via non-random rearrangement in somatic hybrids ${ }^{17,25,28}$. Previous studies have found putative rearrangement hotspots and specific regions preferentially inherited or eliminated. In addition, ${ }^{29}$ speculated that nonrandom mitochondrial rearrangement determining mitogenome types in somatic hybrids might be related to yield components in potatoes. However, these studies did not provide sequence-level evidence for mitogenome recombination events in somatic hybrids.

In this study, we characterized plastome, mitogenomes, and nuclear ribosomal DNA (nrDNA) of tetraploid potato (S. tuberosum), wild diploid potato (S. commersonii), and their somatic hybrid. The comparative analysis revealed their inheritance pattern and exhibited dynamic multipartite structural variation mediated by recombination events that share unique genes from both species in the somatic hybrid mitogenome.

\section{Materials And Methods}

\section{Plant materials and whole genome sequencing}

The study complies with local and national guidelines. Plants of Solanum tuberosum (accession no. PT56: St), S. commersonii (accession no. Lz3.2: Sc), and their somatic hybrids (accession no. HA06-9: StSc) generated by protoplast fusion ${ }^{42}$ were used for the complete assembly of the mitogenome. All plants were grown and maintained at the Highland Agriculture Research Institute (HARI), RDA, Korea. H.-J. P., J.-H. S., J.-H. C., Y.-E. 
P., J.-G. C., and K.-S. C. prepared voucher specimen and identification. The voucher specimen of Sc, St and StSc was assigned HLP1841, HLP1842, and HLP1843, respectively in Potato Germplasm Center, Korea.

The total genomic DNA was extracted from fresh leaves using DNeasy Plant MiniKit (Qiagen, CA, USA) according to the manufacturer's instructions and examined using NanoDrop (DeNovix, Wilmington, USA) and 2100 Expert Bioanalyzer (Agilent Technologies, USA). Paired-end (PE) genomic libraries were constructed according to the standard Illumina PE protocol and sequenced using an Illumina MiSeq platform (Illumina, San Diego, CA) by Macrogen Biotechnology Center (Marcrogen Inc., Seoul, Korea, http://www.macrogen.com/).

\section{De novo assembly and validation of mitochondrial genomes and 45 S nuclear ribosomal DNA}

Raw PE data of approximately $5.8 \mathrm{~Gb}$ for $S$. tuberosum, $6.6 \mathrm{~Gb}$ for $S$. commersonii, and $5.3 \mathrm{~Gb}$ for somatic hybrid were generated and used for assembly (Table S1). De novo mitogenome assembly was performed using PE data according to a previous study ${ }^{43}$. Briefly, high-quality read sequences (Phred score > 20) were obtained, and de novo assembly was conducted using the clc_novo_assemble tool in the CLC Assembly Cell package (ver. 4.2.1, CLC Inc., Denmark). Contigs derived from raw data of mitogenomes were selected based on similarity with mitogenome sequences of other Solanaceae species, such as Capsicum annuum (GenBank acc. no. KJ865409), and Nicotiana tabacum (GenBank accession No. no. KR780036), and then extended, gap-filled, and merged through a series of read mapping to generate a draft circular mitogenome sequence.

The draft mitogenome sequences were validated using bioinformatics and experimental methods. For validation based on PE read mapping, the high-quality PE reads were mapped again on the draft mitogenome sequences; subsequently, the consistency and connectivity of the mapped reads on draft mitogenomes and on junctions between contigs were confirmed. In read mapping, the coexistence of chloroplast or nuclear genome-derived reads was manually confirmed and removed based on extremely high or low depth.

For validation based on PCR amplification and nucleotide sequencing, specific primers for each subgenome were designed using the NCBI PrimerBLAST tool (https://www.ncbi.nlm.nih.gov/tools/primer-blast/index.cgi) and used for genomic DNA PCR amplification and nucleotide sequencing (Table S2). For PCR analysis, 10 ng of genomic DNA was used in a 20- $\mu \mathrm{L}$ PCR mixture of AccuPower PCR PreMix (Bioneer, Daejeon, Korea) that consists of $0.2 \mathrm{U} / \mu \mathrm{L}$ TOP DNA polymerase, $1.5 \mathrm{mM} \mathrm{Mg}^{2+}$, and $250 \mathrm{uM}$ each dNTP mixture with 5 pmole of each primer. PCR conditions were as follows: $95^{\circ} \mathrm{C}$ for $5 \mathrm{~min} ; 25$ cycles of $95^{\circ} \mathrm{C}$ for $30 \mathrm{~s}, 58^{\circ} \mathrm{C}$ for $30 \mathrm{~s}$, and $72{ }^{\circ} \mathrm{C}$ for 1 min; and $72{ }^{\circ} \mathrm{C}$ for 10 min. PCR products were analyzed by $1.8 \%$ agarose gel electrophoresis. The nucleotide sequences of the PCR amplicons were determined by Sanger sequencing and compared with the draft mitogenome sequence for validation.

\section{Mitochondrial genome annotation}

Mitogenomes were initially annotated using the GeSeq program (https://chlorobox.mpimp-golm.mpg.de/geseq-app.html) ${ }^{44}$, and the genes were further predicted by comparison with mitogenomes of other Solanaceae species such as Capsicum annuum (GenBank acc. no. KJ865409) and Nicotiana tabacum (GenBank accession No. no. KR780036). Ambiguous gene positions were manually corrected using NCBI BLASTN-based search analysis and the Artemis annotation tool ${ }^{45}$. A linear and circular map of the mitogenome with annotation information was drawn using the OGDRAW program (https://chlorobox.mpimp-golm.mpg.de/OGDraw.html) ${ }^{46}$.

Repetitive sequences such as direct and palindrome repeats in the mitogenome were searched using the Vmatch program (http://www.vmatch.de) ${ }^{47}$ integrated with the REPuter program with a minimum repeat length of 20 bp and then selected using a length cut-off value of $100 \mathrm{bp}$. Tandem repeats were searched using the Tandem Repeats Finder program with parameters such as match 2, mismatch 7, indels 7, minimum alignment score 80 , maximum period size 10, and maximum TR size 50 (https://tandem.bu.edu/trf/trf.html) ${ }^{48}$.

\section{Identification of genome collinearity and sequence variation between mitochondrial genomes}

Genome collinearity regions among mitogenomes were identified by reciprocal BLASTN searches with a cutoff e-value of 1e-1 and a minimum length of $1000 \mathrm{bp}$ (Table S3). In addition, the origin of somatic hybrid mitogenome sequences was inferred by comparison with $S$. tuberosum and S. commersonii mitogenome sequences using Mega BLASTN searches with parameters of cut-off e-value $1 \mathrm{e}-1$ and minimum match length 1000 bp. A collinearity map was visualized using a Circos program in R package and OGDRAW program (https://chlorobox.mpimp-

golm.mpg.de/OGDraw.html) ${ }^{46}$. Mitogenome sequences possibly derived from chloroplast and nuclear genomes were identified using BLASTNbased analysis with a cutoff e-value of 1E-1 against $S$. tuberosum chloroplast (GenBank accession no. JF772171) and nuclear genomes (SolTub_3.0, https://www.ncbi.nlm.nih.gov/assembly/GCF_000226075.1/).

\section{Phylogenetic analysis and calculation of nucleotide substitution rate}

The phylogenetic analysis based on the maximum likelihood (ML) was performed using conserved 35 protein-coding gene (PCG) sequences (nad1, nad2, nad3, nad4, nad4L, nad5, nad6, nad7, nad9, sdh3, sdh4, cob, cox1, cox2, cox3, atp1, atp4, atp6, atp8, atp9, ccmB, ccmC, ccmFc, ccmFN, rps3, rps4, rps10, rps12, rps13, rp/2, rp/5, rp/10, rp/16, matR, and $m t t B$ ) in 14 species (13 in Solanaceae and one out group in Oleaceae) 
belonging to the Asterids group (Table S4). Concatenated PCG sequences were aligned using the MAFFT program (ver. 7) ${ }^{49}$. To find the best substitution models, the jModelTest program-based (ver. 2.1.10) analysis was conducted using the Akaike information criterion (AIC), Bayesian information criterion (BIC), and invariable site options ${ }^{50}$. ML phylogenetic trees were constructed, and various programs were compared, such as, RAxML (ver. 8.2.12) ${ }^{51}$, MEGA (ver. 7) ${ }^{52}$, and PhyML (ver. 3) ${ }^{53}$ programs. A Bayesian phylogenetic tree was constructed using the BEAST (ver. 2.6.2) ${ }^{54}$ and MrBayes (ver. 3.2.7) ${ }^{55}$ programs. Finally, the selected phylogenetic analysis was conducted using RAxML (ver. 8.2.12) with the GTR $+\Gamma+$ I (invariable) nucleotide substitution model, 1000 bootstraps, and 1000 random number seed options ${ }^{51}$. A phylogenetic tree was constructed using the FigTree program (ver. 1.4.2, http://tree.bio.ed.ac.uk/software/figtree/). The non-synonymous substitution (Ka) and synonymous substitution (Ks) ratios were calculated for the 35 PCG sequences using the CodeML tool in the PAML software package ${ }^{56}$.

\section{Genomic in situ hybridization (GISH)}

S. tuberosum (accession no. PT56) gDNA $(1 \mu \mathrm{g})$ was labeled with Texas Red-5-dUTP (Perkin Elmer, NEL417001EA) and Alexa Fluor 488-5-dUTP (Invitrogen, C11397), respectively, through direct nick translation. These labeled gDNAs were used as probes for GISH. Slides with metaphase chromosomes of somatic hybrids were fixed with $4 \%$ paraformaldehyde for 5 min, dehydrated in an ethanol series (70\%-100\%), and air-dried. The probe hybridization mixture contained 50\% formamide, 10\% dextran sulfate, 2x SSC, 20 ng of each gDNA probe, and DNase-free water. Probes were denatured at $90^{\circ} \mathrm{C}$ for $10 \mathrm{~min}$ and immediately placed on ice for at least 5 min prior to mounting on slides with good metaphase chromosome spreads. A total volume of $40 \mu \mathrm{L}$ was mounted per slide. Next, chromosomes were co-denatured with the probes at $80{ }^{\circ} \mathrm{C}$ for $3-5$ min on a ThermoBrite (Fisher Scientific, USA) and hybridized overnight at $37^{\circ} \mathrm{C}$. Slides were then washed in $2 x$ SSC for 5 min at RT, $0.1 \times$ SCC for 25 min at $42{ }^{\circ} \mathrm{C}$, and $2 \times$ SSC for 5 min at $20^{\circ} \mathrm{C}$. After ethanol series $(70 \%, 90 \%, 100 \%)$ dehydration for 2 min each, the slides were air-dried and counterstained with $1 \mu \mathrm{g} / \mathrm{mL}$ DAPI in Vectashield (Vector Laboratories, USA). Images were captured using an Olympus BX53 fluorescence microscope equipped with a Leica DFC365 FS CCD camera and processed using Cytovision ver. 7.2 Leica Microsystems, Germany). We performed further image enhancements using Adobe Photoshop CC.

\section{Results}

\section{Complete mitochondrial genome assembly}

The mitogenomes of St, Sc, and StSc were assembled into five to two subgenomes through de novo assembly using 5.3 to 6.6 Gb PE reads. Each assembly was validated by conducting PCR analysis and sequencing (Tables S1 and S2, Figure S1). The St mitogenome size was 756,058 bp, and it was composed of five circular subgenomes of lengths 49,230 to 297,014 bp. The total number of non-redundant genes was 78 , consisting of 37 PCGs, 19 ORFs, 3 rRNAs, and 19 tRNAs (Table 1, Figure S2A). The Sc mitogenome was 552,103 bp in size with two subgenomes (338,427 and 213,676 bp). The total number of non-redundant genes was 77, consisting of 37 PCGs, 20 ORFs, 3 rRNAs, and 17 tRNAs (Table 1, Figure S2B). The StSc mitogenomes were 447,645 bp in size with a major circular DNA of 398,439 bp and a minor subgenome of 49,206 bp. The total number of non-redundant genes was 77, consisting of 37 PCGs, 20 ORFs, 3 rRNAs, and 17 tRNAs (Table 1, Figure S2C).

A total of 71 genes were shared among the three mitogenomes. Some genes were unique in each mitogenome: four ORFs (orf131, orf 190, orf 240, and orf 279), three tRNAs (trnl-GAU, trnL-CAA, and trnV-GAC) were unique in the St mitogenome; five ORFs (orf109d, orf111, orf140, orf185, orf240) and one tRNA (trnfM-CAT) were unique in the Sc genome; and four ORFs (orf111, orf127, orf131, orf140, orf185) and one tRNA (trnV-GAC) were unique in the StSc mitogenome (Table 2).

\section{Mitogenome homologs in plastome and nuclear genome}

Mitochondrial plastid DNA (MTPT) has been reported in various plants, such as Amborella trichopoda, Zea mays (maize), and Cynanchum wilfordii. ${ }^{30-32}$. The degree of MTPT was examined by sequence comparison with the $S$. tuberosum plastome sequence (GenBank accession No. no. KM489056). Consequently, the St, Sc, and StSc mitogenomes were approximately 1.0-8.0 \%, 2.9-8.0 \%, and 3.1-4.0 \% considered as MTPT, respectively. Overall, approximately $1.0 \%-2.0 \%$ were identified as MTPT (Table 1, Figures S2).

Further, nuclear mitochondrial DNA (NUMT) has also been reported in various plants, such as Arabidopsis thaliana and Cucumis sativus (cucumber) ${ }^{33,34}$. NUMT was identified by sequence comparison with the $S$. tuberosum nuclear genome sequence (SolTub_3.0, https://www.ncbi.nlm.nih.gov/assembly/GCF_000226075.1/). Consequently, the St, Sc, and StSc mitogenomes were approximately 17.2-57.7 \%, $16.1-17.4 \%$, and 10.1-16.3\%, which were considered to be derived from or transferred to nuclear genomes accordingly. Overall, approximately 10.7 $\%-10.7 \%$ was identified as NUMTs. A total of $57.7 \%$ was identified in St subgenome 4 , which has a very small genome size (Table 1 , Figures S2).

\section{Homologous recombination mediated by large repeats in mitogenomes}

Homologous recombination (HR) can be mediated by repeat sequences in St, Sc, and StSc mitogenomes. The St, Sc, and StSc mitogenomes accounted for approximately $2.2-19.4 \%, 4.8-21.3 \%$, and $5.7-25.9 \%$ of repeat sequences in which the repeat ratio was also positively correlated with the subgenome size (Table 1, Figures 1 and S2). The five St subgenomes exhibited diverse numbers of dispersed repeats: 300 (mitogenome 
coverage: $19.4 \%), 211$ (15.2\%), 41 (5.5\%), 18 (2.2 \%), and 39 (4.9\%) in each subgenome (Tables 1 and S5, Figures 1A and S2A). The two Sc subgenomes included 460 (25.9\%) and 198 dispersed repeats (15.2\%) (Tables 1 and S5, Figures 1B and S2B). Further, the two StSc subgenomes contained 480 (21.3\%) and 39 (4.8\%) dispersed repeats (Tables 1 and S5, Figure 1C and S2C). In contrast, tandem repeats were selected with adjacent sequences of at least two copies and up to $50 \mathrm{bp}$. The St, Sc, and StSc mitogenomes had only 26, 20, and 16 tandem repeats, respectively (Table S6).

Two large repeats (more than 1kb) were identified in the St subgenome 1. R1 was 11916 bp, and R2 was 7500 bp. In contrast, St subgenome 2 had only R1, and subgenome 3 had only 1,589 bp of R3. Similarly, the R1 sequence co-existed in St subgenomes 1 and 2 . The R2 repeat is shared between subgenomes 1 and 4 (Table S5, Figures 1 and S2), which might contribute to the HR between different subgenomes. The Sc mitogenomes had two multipartite structures, in which three large repeats of more than 1kb were identified (R1: 16,857 bp, R2: 10,094 bp, and R3: 1,024 bp), which might contribute to recombination events between subgenomes (Table S5, Figures 1 and S2). The StSc mitogenomes contain four large repeats (more than 1kb) (R1, 11,916 bp; R2, 11,846 bp; R3, 1,643 bp; and R4, 1,024 bp) that might contribute to subgenome reshuffling (Table S5, Figures 1 and S2).

\section{Confirmation of the somatic hybrid in mitochondria and nuclear genomes}

We compared plastomes, mitogenomes, and nrDNAs among St, Sc, and StSc genomes. The StSc plastome was identical to Sc plastome. Meanwhile, the StSc mitogenome shows a complicated structure with unique genes derived from both species (Table S3, Figures 2 ). Among 71 common genes, 21 PCGs (nad3, nad4, nad4L, nad5, nad6, sdh3, cox2, cox3, atp1, atp4, atp8, atp9, ccmB, rps3, rps4, rps12, rps13, rp/5, rp/10, $r p / 16$, and $m t t B$ ) were found identical across the three mitogenomes (denoted as green boxes on Figure 2) and their origin in the StSc genome could not be determined; 12 PCGs (nad1, nad2, nad7, nad9, sdh4, cob, cox1, ccmC, ccmFc, rps10, rp/2, matR) were found identical with Sc (represented as sky-blue boxes in Figure 2) and 2 PCGs (atp6 and ccmFN) were identical with St (pink boxes in Figure 2). Therefore, it is likely that the majority of the somatic hybrid mitogenomes originated from Sc (Figure 2).

GISH data using Sc genome probes revealed strong signals in 24 chromosomes but weak signals in the other 24 chromosomes in the StSc somatic hybrid (Figure $3 \mathrm{~A}$ ). The analysis of the 45S nrDNA in the StSc genome revealed both genotypes of St and Sc with overall mapping depth of 0.7 and $0.3 \%$ for Sc and St, respectively (Figure $3 B$ ).

In summary, St, a dihaploid of tetraploid cultivated potato, has five mitogenomes. Sc, a diploid wild potato, has two mitogenomes. Somatic hybrids developed via protoplast fusion of these two diploids contain the Sc-unique plastome but recombined mitogenomes and nuclear genomes derived from both St and Sc genomes (Figure 4).

\section{Mutation rate of mitochondrial genes in Solanaceae}

A total of 35 PCGs were common across Solanaceae. The nonsynonymous substitution (Ka), synonymous substitution (Ks), and their ratios were calculated. The Ka values ranged from 0 to 0.119 with a 0.003 of median value. The nad4 and nad4L genes had the lowest Ka values, while atp6 had the highest Ka value. The Ks values ranged from 0.02 to 0.228 with a 0.01 of median value. Moreover, $m t t B$ and atp 6 had the lowest and highest Ks values, respectively. Lastly, the Ka/Ks values ranged from 0 to 3.528 with a median value of 0.286 (Table S8, Figure $5 \mathrm{~A}$ ). A Ka/Ks value of more than 2 was observed due to the extremely low Ks value.

Although the Ka and $\mathrm{Ks}$ values were generally low, $c c m F c$ and $m t t B$ exhibited high $\mathrm{Ka} / \mathrm{Ks}$ values of more than 1 , indicating that these genes were positively selected during evolution (Figure $5 \mathrm{~A}$ ). Considering that atp6 showed a high mutation rate above 0.1 . Ka and Ks values relative to the other genes, the amino-acid sequences corresponding to atp6 were compared among Solanaceae species, which revealed that amino acid sequences were variable at the $\mathrm{N}$-terminus but conserved at the $\mathrm{C}$-terminus. (Figure 5B).

Phylogenetic trees were constructed using various programs, including RAxML, MEGA7, PhyML, and BEAST to examine the topology of the species. Trees treated with RAXML, PhyML, and BEAST displayed the same topology, while those treated with MEGA7 exhibited slightly different topologies (Figure S3). In trees generated using RAxML representing an optimized topology (Figures 6 and S3), Solanaceae species were divided into two subfamilies, Solanoideae and Nicotianoideae, and the somatic hybrid exhibited a moderate branch between St and Sc. During the evolution of Solanaceae mitogenome, first, rps1 and rps19 were present in Solanaceae, however, these were omitted completely in Oleaceae. Next, rps7 was confirmed to be completely deleted in Solanaceae compared to Oleaceae. Lastly, ycf14 in all Nicotianoideae species was pseudogenized in the divergence period between Solanoideae and Nicotianoideae (Figure 6).

\section{Discussion}

\section{Diverse mitogenome structures in potato}

The potato ( $S$. tuberosum) nuclear genome was reported by the Potato Genome Sequencing Consortium ${ }^{35}$, and mitogenomes were reported by our group for S. tuberosum (MF989953-MF989957) and S. commersonii (MF989960-MF989961) ${ }^{16}$. In this study, we sequenced a novel 
mitogenome for somatic hybrids (MF989958-MF989959) of St and Sc and conducted a comparative genome analysis. These assemblies play an important role in the unique genetic inheritance patterns of somatic hybrid mitogenomes. Plant mitogenomes exhibit dynamic recombinant structures mediated by HR ${ }^{36,37}$. The large repeats shared in St, Sc, and StSc mitogenomes might facilitate HR events in St, Sc, and StSc.

The HR of mitogenomes mediated by dispersed repeats was suggested to consist of a multivariate configuration and subgenomes ${ }^{38}$. Similar to the five sub-mitogenomes of S. tuberosum (accession no. PT56: St), another mitogenome was reported using the cultivar Cicero and Désirée (hereafter referred to as Cicero) based on PacBio sequencing ${ }^{39}$. Meanwhile, our assembled and Cicero mitogenomes exhibited noticeable differences. The largest mitogenome of Cicero is the fused form of mitogenome subgenomes 1, 2, and 4 (Figure S5). Our mitogenome subgenome-1 was assembled in a circular form; in contrast, the Cicero's largest mitogenome was observed in the linear form. The St submitogenomes 1, 2, and 4 shared large repeats that could mediate HR (Figures 1 and 4), suggesting that various sub-mitogenomes can exist in different individuals or tissues.

Furthermore, atp6 in St sub-mitogenome 1 was prematurely terminated as 321 amino acids (aa) in length, whereas the Cicero mitogenome had a full structure gene of 389 aa. The discrepancy of atp 6 was examined thoroughly, and a linear fragment with a complete structure of 389 aa was found by mapping to atp6 sequence of Cicero mitogenome. This suggests that an unrevealed linear form or short fragment could also contain essential genes in the mitogenome, such as atp6, even if not in the circular form.

\section{Transmission of plastome and mitogenomes in somatic hybrid}

The chloroplasts of somatic hybrids were randomly selected and delivered from parents, and the nuclei or mitochondria are known to fuse ${ }^{9}$. The StSc plastome was transferred only from S. cerevisiae ${ }^{40}$. In addition, StSc mitogenomes were randomly rearranged between St and Sc in most regions. The clustering analysis of CDSs revealed that the majority of the genes were derived from Sc; however, certain genes were derived from St (Figure 2).

Previous studies have suggested that the somatic hybrids harbor recombinant mitogenomes that share both mitogenome types based on fingerprinting patterns ${ }^{9,20,28,29}$. In this study, we have displayed sequence-level recombination events that share unique genes from each of the parental species of somatic hybrids. Although this phenomenon may have occurred entirely randomly, fundamental mitochondrial genes in the Solanaceae family were preserved. Although the recombination mechanism is unclear, we assume that the smaller mitogenome might be competitive during somatic hybridization.

\section{Low evolutionary rate of mitochondrial genes in Solanaceae}

To date, missing or misnamed genes have been examined in the Solanaceae mitogenome, and it has been confirmed that 35 PCGs are commonly preserved (Table S7). These genes were found to have few mutations, and even if mutations existed, most of them were identified as synonymous substitutions. This result is consistent with the fact that mitogenomes have few gene variations ${ }^{33,41}$. Exceptionally, atp6 exhibited large length variation. Moreover, atp6 was present in an intact form with a short length; however, the Solanaceae mitogenome evolutionary process confirmed that the sequence continued to accumulate in the front of the conserved motif (Figure 5). It was considered that atp6 could continuously promote mutations, suggesting that it can be useful for Solanaceae DNA barcoding. Novel ORFs that might be created during somatic hybridization have been identified; however, their function is unknown.

Based on the NCBI land plant organelle database (https://www.ncbi.nlm.nih.gov/genome/organelle/), $5295 \mathrm{cp}$ genomes have been published, whereas only 279 mitogenomes have been released (July 2021). This can be attributed to the difficulty in assembling the mitogenome compared to the plastome. Therefore, our phylogenetic study will play an important role in identifying the relationship during mitochondrial evolution. Our phylogenetic trees were slightly different from those of traditional plastome-based trees ${ }^{16}$. The Solanum, Capsicum, and Nicotiana genera were assigned to the same lineage. However, in all other phylogenetic trees, Capsicum was grouped with S. Iycopersicum and S. pennelii. This was due to the low mutation rate of the mitogenome.

\section{Declarations}

\section{Data Accessibility}

The complete mitogenome have been deposited in the National Center for Biotechnology Information (NCBI) database under GenBank accession numbers MF989958-MF989959 for Somatic Hybrid, MF989960-MF989961 for S. commersonii, and MF989953-MF989957 for S. tuberosum.

\section{Author contributions}

H.-O. L., T.-J. Y., and K.-S. C. designed research; H.-J. P., J.-H. S., J.-H. C., Y.-E. P., J.-G. C., and K.-S. C. performed sample preparation; H.-O. L., S.-C. L., and K.-S. C. analyzed data; H.-O. L., S.-C. L., and K.-S. C. wrote the manuscript; H.-O. L., T.-J. Y., and K.-S. C. revised the manuscript; All authors

Page 6/12 
reviewed the manuscript.

\section{Acknowledgements}

This work was supported by an Agenda Project (No. PJ012540) of Rural Development Administration in South Korea.

\section{References}

1. Birky, C. W. The Inheritance of Genes in Mitochondria and Chloroplasts: Laws, Mechanisms, and Models. Annual Review of Genetics, 35, 125148 https://doi.org/doi:10.1146/annurev.genet.35.102401.090231 (2001).

2. Ahokas, H. Cytoplasmic male sterility in barley: Evidence for the involvement of cytokinins in fertility restoration. Proceedings of the National Academy of Sciences 79, 7605, doi:10.1073/pnas.79.24.7605 (1982)

3. Fauron, C., Havlik, M., Lonsdale, D. \& Nichols, L. Mitochondrial genome organization of the maize cytoplasmic male sterile type T. Molecular and General Genetics MGG, 216, 395-401 https://doi.org/doi:10.1007/BF00334381 (1989).

4. Bi, C. et al. Analysis of the Complete Mitochondrial Genome Sequence of the Diploid Cotton Gossypium raimondii by Comparative Genomics Approaches. BioMed Research International 2016, 5040598, doi:10.1155/2016/5040598 (2016)

5. Alverson, A. J. et al. Insights into the Evolution of Mitochondrial Genome Size from Complete Sequences of Citrullus lanatus and Cucurbita pepo (Cucurbitaceae). Molecular Biology and Evolution, 27, 1436-1448 https://doi.org/doi:10.1093/molbev/msq029 (2010).

6. Braun, H. P. et al. The life of plant mitochondrial complex I., 19, 295-313 https://doi.org/doi:10.1016/j.mito.2014.02.006 (2014).

7. Mackenzie, S. \& Mclntosh, L. Higher Plant Mitochondria., 11, 571 https://doi.org/doi:10.1105/tpc.11.4.571 (1999).

8. Sloan, D. B., Alverson, A. J., Wu, M., Palmer, J. D. \& Taylor, D. R. Recent Acceleration of Plastid Sequence and Structural Evolution Coincides with Extreme Mitochondrial Divergence in the Angiosperm Genus Silene. Genome Biology and Evolution, 4, 294-306 https://doi.org/doi:10.1093/gbe/evs006 (2012).

9. Guri, A. \& Sink, K. C. Interspecific somatic hybrid plants between eggplant (Solanum melongena) and Solanum torvum. Theoretical and Applied Genetics, 76, 490-496 https://doi.org/doi:10.1007/BF00260897 (1988).

10. Noyszewski, A. K. et al. Accelerated evolution of the mitochondrial genome in an alloplasmic line of durum wheat. BMC Genomics, 15,67 https://doi.org/doi:10.1186/1471-2164-15-67 (2014).

11. Michalovova, M., Vyskot, B. \& Kejnovsky, E. Analysis of plastid and mitochondrial DNA insertions in the nucleus (NUPTs and NUMTs) of six plant species: size, relative age and chromosomal localization., 111, 314-320 https://doi.org/doi:10.1038/hdy.2013.51 (2013).

12. Ogihara, Y. et al. Structural dynamics of cereal mitochondrial genomes as revealed by complete nucleotide sequencing of the wheat mitochondrial genome. Nucleic Acids Res, 33, 6235-6250 https://doi.org/doi:10.1093/nar/gki925 (2005).

13. Jo, Y. D., Choi, Y., Kim, D. H., Kim, B. D. \& Kang, B. C. Extensive structural variations between mitochondrial genomes of CMS and normal peppers (Capsicum annuum L.) revealed by complete nucleotide sequencing. BMC Genomics, 15, 561 https://doi.org/doi:10.1186/1471-216415-561 (2014).

14. Cho, K. S. et al. The complete mitochondrial genome sequences of potato (Solanum tuberosum L., Solanaceae). Mitochondrial DNA Part B, 2 , 781-782 https://doi.org/doi:10.1080/23802359.2017.1398607 (2017).

15. Kim, H. T. \& Lee, J. M. Organellar genome analysis reveals endosymbiotic gene transfers in tomato. PLOS ONE, 13, e0202279 https://doi.org/doi:10.1371/journal.pone.0202279 (2018).

16. Cho, K. S. et al. Mitochondrial genome sequence of tuber-bearing wild potato, Solanum commersonii Dunal. Mitochondrial DNA Part B, 3 , 198-199 https://doi.org/doi:10.1080/23802359.2018.1437826 (2018).

17. Temple, M., Makaroff, C. A., Mutschler, M. A. \& Earle, E. D. Novel mitochondrial genomes in Brassica napus somatic hybrids. Curr. Genet, 22, 243-249 https://doi.org/doi:10.1007/BF00351732 (1992).

18. Wachocki, S. E., Bonnema, A. B. \& O'Connell, M. A. Comparison of the organization of the mitochondrial genome in tomato somatic hybrids and cybrids. Theoretical and Applied Genetics, 81, 420-427 https://doi.org/doi:10.1007/BF00228686 (1991).

19. Arimura, S., Yanase, S., Tsutsumi, N. \& Koizuka, N. The mitochondrial genome of an asymmetrically cell-fused rapeseed, Brassica napus, containing a radish-derived cytoplasmic male sterility-associated gene. Genes \& Genetic Systems advpub, doi:10.1266/ggs.18-00005 (2018)

20. Smyda, P. et al. Development of somatic hybrids Solanum $\times$ michoacanum Bitter. (Rydb.) (+) S. tuberosum L. and autofused $4 \times S$. $\times$ michoacanum plants as potential sources of late blight resistance for potato breeding. Plant Cell Rep, 32, 1231-1241 https://doi.org/doi:10.1007/s00299-013-1422-5 (2013).

21. Thieme, R. et al. Novel somatic hybrids (Solanum tuberosum L. + Solanum tarnii) and their fertile BC1 progenies express extreme resistance to potato virus $Y$ and late blight. Theoretical and Applied Genetics, 116, 691 https://doi.org/doi:10.1007/s00122-007-0702-2 (2008).

22. Greplova, M. et al. Characterization of somatic hybrids Solanum bulbocastanum+ dihaploid Solanum tuberosum.Acta horticulturae(2012)

Page $7 / 12$ 
23. lovene, M. et al. Nuclear and cytoplasmic genome composition of Solanum bulbocastanum (+) S. tuberosum somatic hybrids. Genome, $\mathbf{5 0}$, 443-450 https://doi.org/doi:10.1139/G07-024 (2007).

24. Cardi, T., Bastia, T., Monti, L. \& Earle, E. D. Organelle DNA and male fertility variation in Solanum spp. and interspecific somatic hybrids. Theoretical and Applied Genetics, 99, 819-828 https://doi.org/doi:10.1007/s001220051301 (1999).

25. Kemble, R. J., Barsby, T. L., Wong, R. S. C. \& Shepard, J. F. Mitochondrial DNA rearrangements in somatic hybrids of Solanum tuberosum and Solanum brevidens. Theoretical and Applied Genetics, 72, 787-793 https://doi.org/doi:10.1007/BF00266546 (1986).

26. Chen, L. et al. Nuclear and cytoplasmic genome components of Solanum tuberosum + S. chacoense somatic hybrids and three SSR alleles related to bacterial wilt resistance. Theoretical and Applied Genetics, 126, 1861-1872 https://doi.org/doi:10.1007/s00122-013-2098-5 (2013).

27. Nouri-Ellouz, O. et al. Production of potato intraspecific somatic hybrids with improved tolerance to PVY and Pythium aphanidermatum. Journal of Plant Physiology, 163, 1321-1332 https://doi.org/doi:10.1016/j.jplph.2006.06.009 (2006).

28. Landgren, M. \& Glimelius, K. Analysis of chloroplast and mitochondrial segregation in three different combinations of somatic hybrids produced within Brassicaceae. Theoretical and Applied Genetics, 80, 776-784 https://doi.org/doi:10.1007/BF00224191 (1990).

29. Lössl, A., Frei, U. \& Wenzel, G. Interaction between cytoplasmic composition and yield parameters in somatic hybrids of $S$. tuberosum L. Theoretical and Applied Genetics, 89, 873-878 https://doi.org/doi:10.1007/BF00224512 (1994).

30. Park, H. S. et al. Mitochondrial plastid DNA can cause DNA barcoding paradox in plants. Sci. Rep, 10, 6112 https://doi.org/doi:10.1038/s41598-020-63233-y (2020).

31. Gandini, C. L. \& Sanchez-Puerta, M. V. Foreign Plastid Sequences in Plant Mitochondria are Frequently Acquired Via Mitochondrion-toMitochondrion Horizontal Transfer. Sci. Rep, 7, 43402 https://doi.org/doi:10.1038/srep43402 (2017).

32. Stern, D. B. \& Lonsdale, D. M. Mitochondrial and chloroplast genomes of maize have a 12-kilobase DNA sequence in common., 299, 698-702 https://doi.org/doi:10.1038/299698a0 (1982).

33. Alverson, A. J., Rice, D. W., Dickinson, S., Barry, K. \& Palmer, J. D. Origins and Recombination of the Bacterial-Sized Multichromosomal Mitochondrial Genome of Cucumber., 23, 2499 https://doi.org/doi:10.1105/tpc.111.087189 (2011).

34. Stupar, R. M. et al. Complex mtDNA constitutes an approximate 620-kb insertion on Arabidopsis thaliana chromosome 2: Implication of potential sequencing errors caused by large-unit repeats. Proceedings of the National Academy of Sciences 98,5099 , doi:10.1073/pnas.091110398 (2001)

35. Xu, X. et al. Genome sequence and analysis of the tuber crop potato., 475, 189-195 https://doi.org/doi:10.1038/nature10158 (2011).

36. Sanchez-Puerta, M. V., Zubko, M. K. \& Palmer, J. D. Homologous recombination and retention of a single form of most genes shape the highly chimeric mitochondrial genome of a cybrid plant. New Phytol, 206, 381-396 https://doi.org/doi:10.1111/nph.13188 (2015).

37. Arrieta-Montiel, M. P., Shedge, V., Davila, J., Christensen, A. C. \& Mackenzie, S. A. Diversity of the Arabidopsis Mitochondrial Genome Occurs via Nuclear-Controlled Recombination Activity., 183, 1261-1268 https://doi.org/doi:10.1534/genetics.109.108514 (2009).

38. Fauron, C., Casper, M., Gao, Y. \& Moore, B. The maize mitochondrial genome: dynamic, yet functional. Trends in Genetics, 11, 228-235 https://doi.org/doi:10.1016/S0168-9525(00)89056-3 (1995).

39. Varré, J. S. et al. Complete Sequence, Multichromosomal Architecture and Transcriptome Analysis of the Solanum tuberosum Mitochondrial Genome. International Journal of Molecular Sciences, 20, https://doi.org/doi:10.3390/ijms20194788 (2019).

40. Cho, K. S. et al. Complete chloroplast genome sequences of Solanum commersonii and its application to chloroplast genotype in somatic hybrids with Solanum tuberosum. Plant Cell Rep, 35, 2113-2123 https://doi.org/doi:10.1007/s00299-016-2022-y (2016).

41. Arrieta-Montiel, M. P. \& Mackenzie, S. A. in Plant Mitochondria (ed Frank Kempken) 65-82 (Springer New York, 2011)

42. Kim-Lee, H., Moon, J. S., Hong, Y. J., Kim, M. S. \& Cho, H. M. Bacterial wilt resistance in the progenies of the fusion hybrids between haploid of potato and Solanum commersonii. American Journal of Potato Research, 82, 129-137 https://doi.org/doi:10.1007/BF02853650 (2005).

43. Lee, H. O. et al. Assembly of the Mitochondrial Genome in the Campanulaceae Family Using Illumina Low-Coverage Sequencing. Genes, 9 , https://doi.org/doi:10.3390/genes9080383 (2018).

44. Tillich, M. et al. GeSeq - versatile and accurate annotation of organelle genomes. Nucleic Acids Res, 45, W6-W11 https://doi.org/doi:10.1093/nar/gkx391 (2017).

45. Rutherford, K. et al. Artemis: sequence visualization and annotation., 16, 944-945 https://doi.org/doi:10.1093/bioinformatics/16.10.944 (2000).

46. Lohse, M., Drechsel, O., Kahlau, S. \& Bock, R. OrganellarGenomeDRAW-a suite of tools for generating physical maps of plastid and mitochondrial genomes and visualizing expression data sets. Nucleic Acids Res, 41, W575-W581 https://doi.org/doi:10.1093/nar/gkt289 (2013).

47. Kurtz, S. et al. REPuter: the manifold applications of repeat analysis on a genomic scale. Nucleic Acids Res, 29, 4633-4642 https://doi.org/doi:10.1093/nar/29.22.4633 (2001). 
48. Benson, G. Tandem repeats finder: a program to analyze DNA sequences. Nucleic Acids Res, 27, 573-580 https://doi.org/doi:10.1093/nar/27.2.573 (1999).

49. Katoh, K., Rozewicki, J. \& Yamada, K. D. MAFFT online service: multiple sequence alignment, interactive sequence choice and visualization. Briefings in Bioinformatics, 20, 1160-1166 https://doi.org/doi:10.1093/bib/bbx108 (2019).

50. Darriba, D., Taboada, G. L., Doallo, R. \& Posada, D. jModelTest 2: more models, new heuristics and parallel computing. Nat. Methods, 9, 772772 https://doi.org/doi:10.1038/nmeth.2109 (2012).

51. Stamatakis, A. RAxML version 8: a tool for phylogenetic analysis and post-analysis of large phylogenies., 30, 1312-1313 https://doi.org/doi:10.1093/bioinformatics/btu033 (2014).

52. Kumar, S., Stecher, G. \& Tamura, K. MEGA7: Molecular Evolutionary Genetics Analysis Version 7.0 for Bigger Datasets. Molecular Biology and Evolution, 33, 1870-1874 https://doi.org/doi:10.1093/molbev/msw054 (2016).

53. Guindon, S. et al. New Algorithms and Methods to Estimate Maximum-Likelihood Phylogenies: Assessing the Performance of PhyML 3.0. Syst. Biol, 59, 307-321 https://doi.org/doi:10.1093/sysbio/syq010 (2010).

54. Bouckaert, R. et al. BEAST 2.5: An advanced software platform for Bayesian evolutionary analysis. PLOS Computational Biology, 15, e1006650 https://doi.org/doi:10.1371/journal.pcbi.1006650 (2019).

55. Ronquist, F. \& Huelsenbeck, J. P. MrBayes 3: Bayesian phylogenetic inference under mixed models., 19, 1572-1574 https://doi.org/doi:10.1093/bioinformatics/btg180 (2003).

56. Yang, Z. PAML 4: Phylogenetic Analysis by Maximum Likelihood. Molecular Biology and Evolution, 24, 1586-1591 https://doi.org/doi:10.1093/molbev/msm088 (2007).

\section{Tables}

Table 1. Mitogenome features of S. tuberosum, S. commersonii, and their somatic hybrid 


\begin{tabular}{|c|c|c|c|c|c|c|c|c|c|}
\hline \multirow{2}{*}{$\begin{array}{l}\text { Scientific } \\
\text { name } \\
\text { Subgenomes }\end{array}$} & \multicolumn{2}{|c|}{ Somatic Hybrid } & \multicolumn{2}{|c|}{ S. commersonii } & \multicolumn{5}{|c|}{ S. tuberosum } \\
\hline & 1 & 2 & 1 & 2 & 1 & 2 & 3 & 4 & 5 \\
\hline $\begin{array}{l}\text { Genome } \\
\text { length (bp) }\end{array}$ & 398,439 & 49,206 & 338,427 & 213,676 & 297,014 & 247,843 & 112,800 & 49,171 & 49,230 \\
\hline $\begin{array}{l}\text { No. of total } \\
\text { genes (non- } \\
\text { redundant) }\end{array}$ & 77 & & 77 & & 78 & & & & \\
\hline $\begin{array}{l}\text { No. of } \\
\text { protein } \\
\text { coding } \\
\text { genes }\end{array}$ & 37 & & 37 & & 37 & & & & \\
\hline $\begin{array}{l}\text { No. of } \\
\text { hypothetical } \\
\text { genes }\end{array}$ & 20 & & 20 & & 19 & & & & \\
\hline $\begin{array}{l}\text { No. of } \\
\text { ribosomal } \\
\text { RNA genes }\end{array}$ & 3 & & 3 & & 3 & & & & \\
\hline $\begin{array}{l}\text { No. of } \\
\text { transfer RNA } \\
\text { genes }\end{array}$ & 17 & & 17 & & 19 & & & & \\
\hline $\begin{array}{l}\text { No. of total } \\
\text { genes by } \\
\text { subgenome }\end{array}$ & 68 & 14 & 64 & 48 & 49 & 30 & 22 & 12 & 13 \\
\hline $\begin{array}{l}\text { No. of } \\
\text { protein } \\
\text { coding } \\
\text { genes }\end{array}$ & 32 & 6 & 31 & 19 & 20 & 19 & 12 & 5 & 6 \\
\hline $\begin{array}{l}\text { No. of } \\
\text { hypothetical } \\
\text { genes }\end{array}$ & 19 & 3 & 18 & 14 & 15 & 0 & 4 & 2 & 2 \\
\hline $\begin{array}{l}\text { No. of } \\
\text { ribosomal } \\
\text { RNA genes }\end{array}$ & 3 & 0 & 2 & 3 & 3 & 3 & 1 & 0 & 0 \\
\hline $\begin{array}{l}\text { No. of } \\
\text { transfer RNA } \\
\text { genes }\end{array}$ & 14 & 5 & 13 & 12 & 11 & 8 & 5 & 5 & 5 \\
\hline $\begin{array}{l}\text { Proportion of } \\
\text { MTPT (\%) }\end{array}$ & 2.9 & 8.0 & 3.1 & 4.0 & 1.5 & 1.0 & 5.0 & 4.3 & 8.0 \\
\hline $\begin{array}{l}\text { Proportion of } \\
\text { NUMT (\%) }\end{array}$ & 16.1 & 17.4 & 16.3 & 10.1 & 24.1 & 17.5 & 20.0 & 57.7 & 17.2 \\
\hline $\begin{array}{l}\text { Proportion of } \\
\text { repeat } \\
\text { sequence } \\
\text { (\%) }\end{array}$ & 21.3 & 4.8 & 25.9 & 5.7 & 19.4 & 15.2 & 5.5 & 2.2 & 4.9 \\
\hline $\begin{array}{l}\text { GenBank } \\
\text { accession } \\
\text { nos. }\end{array}$ & MF989958 & MF989959 & MF989960 & MF989961 & MF989953 & MF989954 & MF989955 & MF989956 & MF989957 \\
\hline
\end{tabular}

Table 2. Common and unique mitogenome genes of a somatic hybrid and its parent species 


\begin{tabular}{|c|c|c|c|c|}
\hline \multirow[t]{2}{*}{ Group of genes } & \multirow[t]{2}{*}{ Common genes } & \multicolumn{3}{|l|}{ Unique genes } \\
\hline & & Somatic hybrid & S. commersonii & S. tuberosum \\
\hline Complex I & $\operatorname{nad} 1,2,3,4,4 L, 5,6,7,9$ & & & \\
\hline Complex II & $s d h 3, s d h 4$ & & & \\
\hline Complex III & $c o b$ & & & \\
\hline Complex IV & $\cos 1,2,3$ & & & \\
\hline Complex V & atp1, 4, 6, 8(orfB), 9 & & & \\
\hline Cytochrome $\mathrm{c}$ biogenesis & $c c m B, c c m C, c c m F c, c c m F n$ & & & \\
\hline $\begin{array}{l}\text { Large subunit ribosomal } \\
\text { proteins }\end{array}$ & $r p / 2,5,10,16$ & & & \\
\hline $\begin{array}{l}\text { Small subunit ribosomal } \\
\text { proteins }\end{array}$ & $r p s 1,3,4,10,12,13,19$ & & & \\
\hline Maturase & matR & & & \\
\hline Transferase & $m t t B(o r f X)$ & & & \\
\hline Ribosomal RNAs & $r r n 5, r r n 18, r r n 26$ & & & \\
\hline Hypothetical genes & $\begin{array}{l}\text { orf100, 101, 102, 103, 104, } \\
105,108,109,110,122, \\
123,125,138,169,261\end{array}$ & $\begin{array}{l}\text { orf1111, 127, 131, 140, } \\
185\end{array}$ & $\begin{array}{l}\text { orf109d, 111, 140, 185, } \\
240\end{array}$ & $\begin{array}{l}\text { orf131, 190, 240, } \\
279\end{array}$ \\
\hline Transfer RNAs & $\begin{array}{l}\operatorname{trnC} C-G C A, \operatorname{trn} D-G U C, \operatorname{trn} E- \\
U U C, \\
\operatorname{trn} F-G A A, \operatorname{trn} G-G C C, \operatorname{trn} H- \\
G U G, \\
\operatorname{trnK}-U U U, \operatorname{trnM}-C A U, \operatorname{trn} N- \\
G U U, \\
\operatorname{trn} P-U G G, \operatorname{trn} Q-U U G, \operatorname{trn}- \\
G C U, \\
\operatorname{trnS}-G G A, \operatorname{trn} S-U G A, \operatorname{trn} W- \\
C C A, \\
\operatorname{trn} Y-G U A\end{array}$ & $\operatorname{trn} V-G A C$ & $\operatorname{trnfM}-C A T$ & $\begin{array}{l}\operatorname{trn} I-G A U, \\
\operatorname{trn} L-C A A \\
\operatorname{trn} V-G A C\end{array}$ \\
\hline No. of genes & 71 & 6 & 6 & 7 \\
\hline
\end{tabular}

\section{Figures}

\section{Figure 1}

Chord diagram of three Solanum mitogenomes. (A) to (C) represent the homologous regions of the subgenomes. R1 to R3 represent the large repeats that might cause homologous recombination among the corresponding subgenomes. St: S. tuberosum accession no. PT56, Sc: S. commersonii accession no. Lz3.2, StSc: somatic hybrid accession no. HA06-9. 

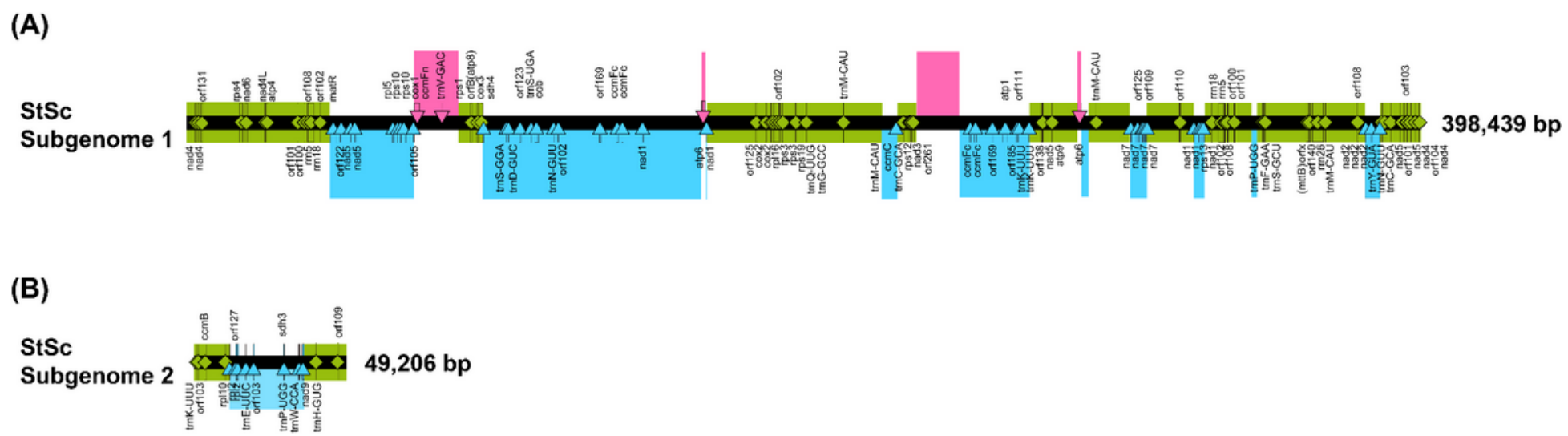

Figure 2

The origin of mitogenome recombination block in somatic hybrid (StSc) (A) Subgenome 1 of somatic hybrid mitogenome (B) Subgenome 2 of somatic hybrid mitogenome. The pink and sky-blue triangles on the black middle line indicate genes derived from $\mathrm{S}$. tuberosum and $\mathrm{S}$. commersonii, respectively. The green diamond boxes indicate genes of unknown origin.

\section{Figure 3}

Detection of nuclear genome fusion in somatic hybrid. (A) GISH analysis of somatic hybrid (HA06-1 clone) using S. tuberosum specific-probes. The red signal of 24 arrows indicates the S. commersonii nuclear subgenomic distribution. (B) Schematic diagram of $45 S$ ribosomal DNA cistron of Solanum species. StSc summary represents the percentage of St or Sc genotypes in the 45SnrDNA sequence.

\section{Figure 4}

Schematic diagram of mitogenome in parental species and their somatic hybrids. (A) S. tuberosum (St), (B) S. commersonii (Sc), and (C) somatic hybrid (StSc). S. tuberosum and S. commersonii have five and two subgenomes, respectively, which are fused into two subgenomes in the somatic hybrid generated by protoplast fusion. The origin of chloroplast genome in somatic hybrid has been determined based on sequence comparison among chloroplast genome sequences of parental species and that of the somatic hybrid.

\section{Figure 5}

Mitochondrial gene diversity in Solanaceae family. (A) non-synonymous substitution (Ka) and synonymous substitution (Ks) values among the 12 Solanaceae species. Ka and Ks values were calculated with 35 protein-coding genes by CodeML program. (B) Variations of atp6 are shown by the phylogenetic tree and multiple comparisons of amino acid sequences. The conserved domain has been determined through NCBI BLASTP search.

\section{Figure 6}

Phylogenetic relationship of 13 Solanaceae species using 35 protein-coding gene sequences commonly conserved in mitogenomes. The maximum likelihood tree was constructed using RAxML program with $\mathrm{GTR}+\Gamma+\mathrm{I}$ model (based on jModelTest2) and a bootstrapping value of 1000 . The bootstrap value ( $>=0.5$ ) is shown on the node. Deleted genes and pseudogenes specifically within each group in the tree have been also shown by red and black boxes, respectively. Olea europaea in the Oleaceae family has been used as an out-group.

\section{Supplementary Files}

This is a list of supplementary files associated with this preprint. Click to download.

- supplementaryTablesFigures20211202.docx 